\title{
Leve eficacia de los anticolinesterásicos en la enfermedad de Alzheimer
}

\section{Objetivo}

Estimar la eficacia terapéutica y la tolerancia de los anticolinesterásicos habitualmente usados en la Enfermedad de Alzheimer (EA)

\section{Fuente de Datos}

La principal búsqueda fue a través de MEDLINE y EMBASE desde 1980 a Mayo 2002. Se incluyeron exclusivamente trabajos publicados en inglés.

\section{Selección de estudios}

Se incluyeron estudios con pacientes adultos con diagnóstico de EA según criterio estándar, que fueran aleatorizados en grupos paralelos, controlados por placebo y evaluados a doble ciego. Los estudios debían durar por lo menos 12 semanas y en la evaluación, incluir una escala cognitiva validada.

\section{Extracción de datos}

Los resultados de los estudios incluidos fueron evaluados según: a) tasa de respuesta global según escalas "clínicas globales" con una respuesta clínica definida como, por lo menos, una mejoría mínima en el estado general del paciente (no se incluyó como respuesta a la estabilidad clínica); b) tasa de respuesta cognitiva definida por una mejoría de cuatro o más puntos en la escala ADAS-cog; c) tasa de abandono al tratamiento, asociada o no a efectos adversos. Se compararon los resultados entre las diferentes drogas.

\section{Resultados principales}

Se incluyeron 16 estudios, con un total de 5159 pacientes tratados. Se identificaron ocho estudios con donepecilo, tres con rivastigmina y cinco con galantamina.

Eficacia global clínica: en ocho estudios se observó una mejoría mínima o mayor del estado clínico general atribuible al tratamiento del 9\% (IC95\%: 6 a 12\%) y se calculó un número necesario para tratar (NNT) de 12 (IC 95\% 9 a 16).

Eficacia Cognitiva: en los cinco estudios de donde se pudo extraer esta variable (1606 pacientes tratados) se obtuvo una mejoría del 10\% (IC95\%: 4 a 17\%), NNT: 10 (8 a 15).

Seguridad: en comparación al placebo, hubo un 8\% (IC95\%: 5 a $12 \%)$ más de efectos adversos reportados. La tasa de abandono del tratamiento debido al efectos adversos fue 7\% (IC95\%: 3 a $10 \%)$ más en el grupo tratado.

Comparación de drogas: no hubo diferencia en la eficacia entre donepecilo y rivastigmina que fue de $13 \%$ (IC95\%: 8 a 17) y $12 \%$ (IC95\%: 5 a 19) respectivamente. Hubo diferencia significativa en la tasa de respuesta clínica entre las dos anteriores vs. galantamina que fue de 5\% (IC95\%: 1 a 8). La tasa de efectos adversos y de abandono del tratamiento fue menor para donepecilo.

\section{Conclusión}

Los anticolinesterásicos evaluados mejoran la funcionalidad en los pacientes con enfermedad de Alzheimer

Fuente de financiamiento: ninguna

\section{Comentario}

Los estudios previos sobre tratamiento de Alzheimer centran su análisis en los cambios cognitivos evidenciados en la escala ADAS- cog. ${ }^{1}$. Una crítica frecuente a estos trabajos es cómo impacta en la clínica un cambio en el ADAS-cog. El presente trabajo focaliza el análisis en la respuesta clínica global, medida por escalas que toman en cuenta la impresión del médico y del cuidador $^{2}$. Estas escalas probablemente reflejen mejor lo que ocurre en el día a día con este tipo de pacientes. EI NNT encontrado para lo que los autores definen como respuesta mínima o mayor fue de 12, pero si buscamos algo más que un cambio mínimo, el NNT sube a 42, según estimaron los autores. Por otro lado, los efectos adversos causaron la discontinuación del tratamiento en uno de cada 16 pacientes.

Si bien hubo diferencia de eficacia y tasa de efectos adversos entre donepecilo, rivastigmina y galantamina, a favor de los dos primeros, hasta el momento no hay estudios comparativos ("cabeza a cabeza") que permitan sacar conclusiones definitivas.

\section{Conclusiones del comentador}

En forma similar a lo que estamos observando en nuestra práctica clínica, este estudio muestra una leve mejoría o una estabilización clínica de la enfermedad de Alzheimer con anticolinesterásicos. Los efectos adversos observados son mayormente molestias digestivas leves. Si bien éstos son relativamente frecuentes, no hay prácticamente otros efectos adversos serios. Los datos de este trabajo muestran que el donepecilo tiene mejores resultados tanto en eficacia terapéutica como en tolerancia, pero no hay estudios "cabeza a cabeza" que confirmen este punto.

Juan L Bernabó, Daniel Seinhart [ Programa de Medicina Geriátrica. Servicio de Clínica Médica. Hospital Italiano de Buenos Aires]

Bernabó J; Seinhart D. Leve eficacia de los anticolinesterásicos en la enfermedad de Alzheimer. Evid. actual. práct. ambul. 2004;7:107. Comentado de: Efficacy and safety of cholinesterase inhibitors in Alzheimer's disease: a meta-analysis. CMAJ. 2003 Sep 16; 169(6):557-64

\section{Referencias}

1.Trinh NH, Hoblyn J, Mohanty S, Yaffe K. Efficacy of cholinesterase inhibitors in the treatment of neuropsychiatric symptoms and functional impairment in Alzheimer's disease: a metaanalysis. JAMA 2003:289:210-6.

2.Patterson CJ et al. Canadian Consensus Conference on Dementia: a physician's guide to using the recommendations. CMAJ 1999;160(12 Suppl):1738-42. 